\title{
A Novel Non-Stationary Channel Model Utilizing Brownian Random Paths
}

\author{
Alireza Borhani, Matthias Pätzold \\ Faculty of Engineering and Science, University of Agder, 4898 Grimstad, Norway \\ Correspondence: Alireza Borhani, alireza.borhani@uia.no \\ Manuscript communication: received 27 November 2013, accepted 9 January 2014
}

\begin{abstract}
This paper proposes a non-stationary channel model in which real-time dynamics of the mobile station (MS) are taken into account. We utilize Brownian motion (BM) processes to model targeted and non-targeted dynamics of the MS. The proposed trajectory model consists of both drift and random components to capture both targeted and non-targeted motions of the MS. The Brownian trajectory model is then employed to provide a non-stationary channel model, in which the scattering effects of the propagation area are modelled by a non-centred one-ring geometric scattering model. The starting point of the motion is a fixed point in the propagation environment, whereas its terminating point is a random point along a predetermined drift. The drift component can be controlled by a so-called drift parameter. Tracking the MS on the proposed Brownian path allows us to derive the local angles-of-arrival (AOAs) and local angles-of-motion (AOMs), which are expressed by stochastic processes rather than random variables. We compute the first-order densities of the AOA and AOM processes in closed form. The local power spectral density (PSD) of the Doppler frequencies and the autocorrelation function (ACF) of the complex channel gain are also provided. Given a walking speed scenario, the analytical results are demonstrated and explained in depth. It turns out that the proposed Brownian path model results in a non-stationary non-isotropic channel model. The proposed geometry-based channel model is very useful for the performance analysis of mobile communication systems under non-stationary conditions.
\end{abstract}

Keywords- Brownian motion processes, non-stationary channels, targeted motions, non-targeted motions, non-centred onering scattering model.

The material in this paper was presented in part at the 6th International Conference on Advanced Technologies for Communications (ATC'13), Ho Chi Minh City, Vietnam, Oct. 2013, and the World Congress on Engineering and Computer Science (WCECS'13), UC Berkeley, USA, Oct. 2013. The corresponding conference papers received the Best Paper Award from ATC'13 and the Certificate of Merit from WCECS'13.

\section{INTRODUCTION}

Channel modelling is recognized as one of the fundamental tasks in the advancement of communication systems. There exist three major approaches to channel modeling: deterministic, stochastic, and geometrybased [1]. Among them, geometric channel models have been found very suitable for modelling non-stationary environments [1-3]. However, most of the geometric channel models proposed in the literature have been simplified under the assumption that the stochastic radio channel is stationary in time. By considering a very short observation time instant, the $\mathrm{AOM}$ and $\mathrm{AOA}$ at the MS can be assumed as random variables such that the stationarity assumption holds. The one-ring scattering model [4-6] and the unified disk scattering model (UDSM) [7] are two examples chosen from the list of stationary channel models in [8] and [7].

Many empirical and analytical investigations, e.g., [911], however, reveal that the stationarity of the channel is only valid for extremely short travelling distances [12]. The potential suitability of geometric channel models for explaining non-stationary environments [1-3] on the one hand, and the results of real- life measurement campaigns [9-11] on the other hand, encourage us to study geometric channel models under non-stationary conditions.

The number of analytical investigations [13-16] with the focus on the statistical properties of non-stationary channels is very limited. For instance, none of the established geometric scattering models listed in $[7,8]$ has been studied under non-stationary conditions. The only exception is the non-stationary one-ring scattering model studied in [17-19], in which it is assumed that the MS travels along either a straight line [17], or a semi-random path with drift [18], or a totally random path [19]. Accordingly, considering the proposed paths in a ring of scatterers allowed us to propose a nonstationary channel model. A disadvantage of the model in [18] is that the path model has been equipped with a Brownian random component only along one of the axes of the Cartesian coordinate system. This randomness is then mapped to the other axis via the slope of the drift. Therefore, the randomness might disappear if the slope is too high (low).

In this paper, we allay the aforementioned drawback by proposing a path model in which two independent $\mathrm{BM}$ processes are assigned to model the random com- 
ponents of the path along each axis. In this regard, the strength of the random components can be controlled by a simple parameter. We also extend the path model of [18] by equipping the model with a so-called drift parameter. This parameter controls the presence of the deterministic drift components along the axes. Accordingly, the straight (line) path model in [17] is obtained from the proposed model if only the drift components exist, but not the random components; the targeted path model (modified and generalized version of the one in [18]) is attained if both the random and drift components exist; the non-targeted path model in [19] is achieved if the random components exist, but the drift components do not. To capture the scattering effects of the propagation environment, the proposed path is supposed to be surrounded by a ring of uniformly distributed scatterers.

By tracking the MS along the proposed Brownian path, we are able to derive the local AOAs and the local AOMs. These quantities are modelled by stochastic processes instead of random variables. We provide the first-order density of the AOA and AOM processes in closed form. In addition, the local PSD of the Doppler frequencies and ACF of the complex channel gain are computed. Considering the operating frequency of the universal mobile telecommunications system (UMTS), we perform numerical computations for a walking speed scenario. The analytical results are displayed to confirm the non-stationarity of the channel model. It is proved that non-stationarity in time is not in line with the common isotropic propagation assumption on the channel. Furthermore, it is shown that the stationary one-ring scattering model [4-6] can be obtained as a special case of the proposed channel model.

The rest of this paper is organized as follows. BM processes are reviewed briefly in Section 2, whereas Section 3 employs BM processes to provide the Brownian path model. Section 4 presents the propagation scenario by means of the non-centred one-ring scattering model. Section 5 derives the complex channel gain of the proposed channel model. Its statistical properties are investigated in Section 6. Numerical results are provided in Section 7. Eventually, the summary of our findings and the conclusions are drawn in Section 8.

\section{Review of Brownian Motion Processes}

A standard BM process $\{B(t): t \in[0, T]\}$ is a Wiener process with normally and independently distributed increments, satisfying the following conditions:

1) $B(0)=0$.

2) $\forall 0 \leq s<t \leq T$, the increment $B(t)-B(s)$ is a normally distributed random variable with zero mean and variance $t-s$, i.e., $B(t)-B(s) \sim$ $N(0, t-s)$.

3) $\forall 0 \leq s<t<u<v \leq T$, the non-overlapping increments $B(t)-B(s)$ and $B(v)-B(u)$ are statistically independent.

$\mathrm{BM}$ processes have a broad range of applications in different branches of science, such as in economy, med- ical science, and engineering. Stock market analysis, diagnosis imaging, and fractal theory [20] are some examples, which benefit from $\mathrm{BM}$ processes in practice. In mobile ad hoc networks, 2D BM processes known as the random walk mobility model, are also used to model atypical motions of mobile nodes [21]. The mobility model is then used to perform network layer analysis. In addition, three-dimensional BM processes have been used to model fully random motions of mobile users in the Euclidian space [22].

\section{Path Modelling}

In what follows, we utilize the spatial representation of the BM process provided in [18]. This representation enables us to introduce a spatial path model rather than a temporal one. Notice that the spatial representation of the path allows us to consider numerous speed scenarios for the MS.

\subsection{Spatial Representation of Brownian Motion Processes}

We assume that the MS starts its motion from a predefined point with Cartesian coordinates $\left(x_{s}, y_{s}\right)$ in the two-dimensional (2D) plane. Moreover, let the point $\left(x_{d}, y_{d}\right)$ denote a reference point (the potential destination point) along the drift of the path. Following the procedure described in [18], we introduce the spatial stochastic process $B(x)$ over the range $\left[x_{s}, x_{d}\right]$, satisfying the following conditions:

1) $B\left(x_{s}\right)=0$.

2) For all $x_{s} \leq x_{p}<x \leq x_{d}$, the increment $B(x)-$ $B\left(x_{p}\right)$ is a normally distributed random variable with zero mean and variance $x-x_{p}$, i.e., $B(x)-$ $B\left(x_{p}\right) \sim N\left(0, x-x_{p}\right)$.

3) For all $x_{s} \leq x_{p}<x<x_{q}<x_{m} \leq x_{d}$, the non-overlapping increments $B(x)-B\left(x_{p}\right)$ and $B\left(x_{m}\right)-B\left(x_{q}\right)$ are statistically independent.

Computational reasons encourage us to consider the spatial BM process at discrete values of $x$. Accordingly, we define $\Delta x=\left(x_{d}-x_{s}\right) / L$ for some positive integer $L$. We also let $B_{l}^{(x)}$ denote the BM process at $x_{l}=x_{s}+$ $l \Delta x(l=0,1, \ldots, L)$. Referring to the last two conditions of the spatial stochastic process $B(x)$, one can conclude that $B_{l}^{(x)}=B_{l-1}^{(x)}+\Delta B_{l}^{(x)}$, where the increment $\Delta B_{l}^{(x)}$ is a normally distributed random variable of the form $N(0, \Delta x)$.

The same procedure can be followed to obtain the spatial stochastic process $B(y)$ over the range $\left[y_{s}, y_{d}\right]$. Analogously, let $\Delta y=\left(y_{d}-y_{s}\right) / L$ stand for the deterministic increment along the $y$-axis. In addition, let the spatial stochastic process $B_{l}^{(y)}$ denote the BM process at $y_{l}=y_{s}+l \Delta y$. A direct consequence is the equality $B_{l}^{(y)}=B_{l-1}^{(y)}+\Delta B_{l}^{(y)}$ in which $\Delta B_{l}^{(y)}$ follows the Gaussian distribution of the form $N(0, \Delta y)$.

\subsection{The Brownian Path Model}

To model motions of the MS in the 2D plane, we propose a path with deterministic drift components 
along each axis. The fluctuations of the path are modeled by two independent spatial BM processes $B_{l}^{(x)}$ and $B_{l}^{(y)}$ associated with the $x$ - and $y$-axis, respectively. Accordingly, the path $\mathcal{P}$ of the MS is modelled by means of

$$
\mathcal{P}:\left\{\begin{array}{l|l}
\left(x_{l}, y_{l}\right) & \begin{array}{l}
x_{l}=x_{s}+k_{d} l \Delta x+\sigma_{x} B_{l}^{(x)} \\
y_{l}=y_{s}+k_{d} l \Delta y+\sigma_{y} B_{l}^{(y)}
\end{array}
\end{array}\right\}
$$

where $l=0,1, \ldots, L$ is the position index, and the drift parameter $k_{d}$ is to manage the behaviour of the deterministic drift components along each axis. Furthermore, the variable $\sigma_{x}\left(\sigma_{y}\right)$ controls the randomness of the path along the $x$-axis (y-axis). Notice that the randomness of the path $\mathcal{P}$ originates inherently from the randomness of the BM process $B_{l}$. Therefore, the parameters $\sigma_{x}$ and $\sigma_{y}$ provide additional degrees of freedom to magnify (attenuate) the randomness.

In what follows, we study several important special cases, which simplify the model in (1) to the path models proposed in [17-19]. We also remark some potential applications of the proposed path model.

\subsection{Special Cases}

A very simple path model can be obtained from (1) if we set $\sigma_{x}=\sigma_{y}=0$, which ignores the random components of the path. In this case, if the drift components exist, i.e., $k_{d}=1$, then the straight-line path model proposed in [17] can be attained as a special case. This model is useful to explain very smooth paths, beginning from a starting point and ending at a terminating point in the $2 \mathrm{D}$ plane.

The path model of [18] can also be obtained as a special case of the model in (1) if we set $\sigma_{x}=0$ and $k_{d}=1$. In this case, the only random component of the path is along the $y$-axis. The drawback of such a model is that by increasing the slope of the drift, the random component will be faded. Nevertheless, the targeted Brownian path model in (1) has been equipped with two independent random components, i.e., $B_{l}^{(x)}$ and $B_{l}^{(y)}$, describing the fluctuations of the path along each axis. In mobile communications, the proposed path model can be used to explain typical dynamics of users in motion, such as pedestrians walking along a street, but not necessarily along a very smooth path. In vehicular communications, the model can be utilized to describe jittery motions of the vehicle antenna, while the vehicle is moving along a road.

Another important special case is obtained by ignoring the drift components, i.e., $k_{d}=0$. By doing so, the path model in (1) reduces to the non-targeted Brownian path model proposed in [19]. This model is known as the random walk mobility model, which is very useful to explain irregular dynamics of mobile users.

\section{The Propagation Scenario}

We use the non-centred one-ring scattering model [18] to capture the scattering effect caused by randomly distributed scatterers in a propagation area. Figure 1

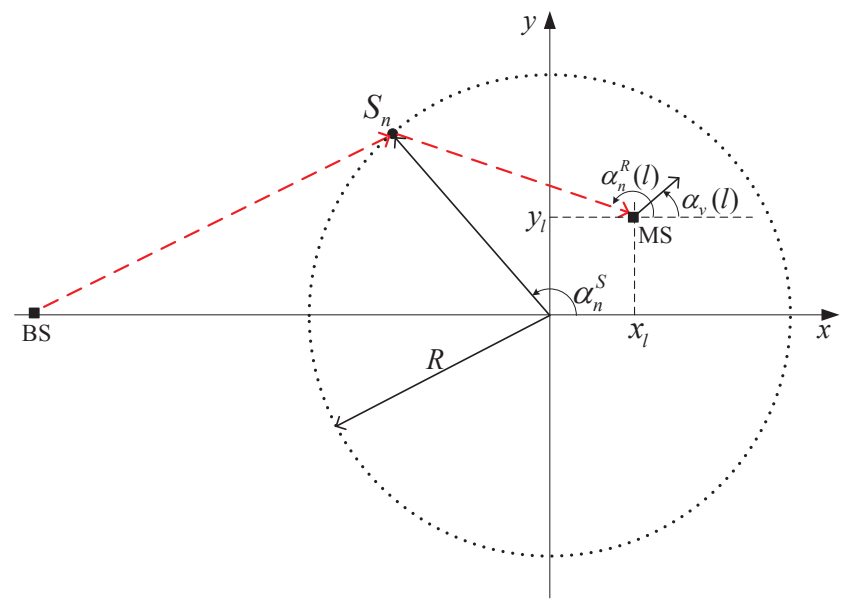

Figure 1. The non-centred one-ring scattering model for a singlebounce scattering scenario.

presents the considered model with the uniform distribution of the local scatterers $S_{n}(n=1,2, \ldots, N)$ on a ring of radius $R$ centered on the origin, but not necessarily on the MS. Referring to this figure, $\boldsymbol{\alpha}_{n}^{S}$ denotes the angle-of-scatterer (AOS) pertinent to the $n$th scatterer $S_{n}$. At a reference point in time $t_{0}$, the MS starts its motion from $\left(x_{0}, y_{0}\right)$ and moves along the path $\mathcal{P}$ to reach the terminating point $\left(x_{L}, y_{L}\right)$ at time $t_{L}$. Subsequently, the position of the MS at time $t_{l} \in\left[t_{0}, t_{L}\right]$ is represented by Cartesian coordinates $\left(x_{l}, y_{l}\right)$. We assume single-bounce scattering, meaning that a wave emitted from the BS arrives at the MS with the AOA $\boldsymbol{\alpha}_{n}^{R}(l)$ after only one bounce due to the $n$th scatterer $S_{n}$. Concerning the velocity scenario, we assume that the MS is in motion with a constant speed of $v_{R}$ in the direction indicated by the AOM $\alpha_{v}(l)$, which is determined by the Brownian path $\mathcal{P}$.

Two realizations of the proposed Brownian path $\mathcal{P}$ in such a geometric scattering model are shown in Figures 2 and 3. It is assumed that the starting point of the path is the origin of the Cartesian system, while the destination point $\left(x_{d}, y_{d}\right)$ is located at $(100,100)$. The radius of the ring has also been set to $R=300 \mathrm{~m}$. Figure 2 displays a realization of the targeted Brownian path in (1) with the parameters $k_{d}=1, L=100$, and $\sigma_{x}=\sigma_{y}=3$. The drift of the path has also been shown by the dotted line. The illustrated path $\mathcal{P}$ varies from realization to realization. However, the general drift of the path does not change. Notice that averaging over different realizations of the targeted path results in a straight line (drift line) from $(0,0)$ to $(100,100)$. Figure 3 exhibits a realization of the non-targeted path $\mathcal{P}$ in (1), where $k_{d}=0, L=100$, and $\sigma_{x}=\sigma_{y}=6$. As it can be observed from this figure, the path exhibits random walk behaviour.

The illustrated propagation scenarios in Figures 1-3 differ completely from the stationary one-ring channel model [4-6], in which the MS is located at the center of the ring of scatterers, while the AOM is a fixed value. Therein, assuming a very short observation time interval justifies a stationary and isotropic channel model, while herein, the proposed path $\mathcal{P}$ results in a non- 


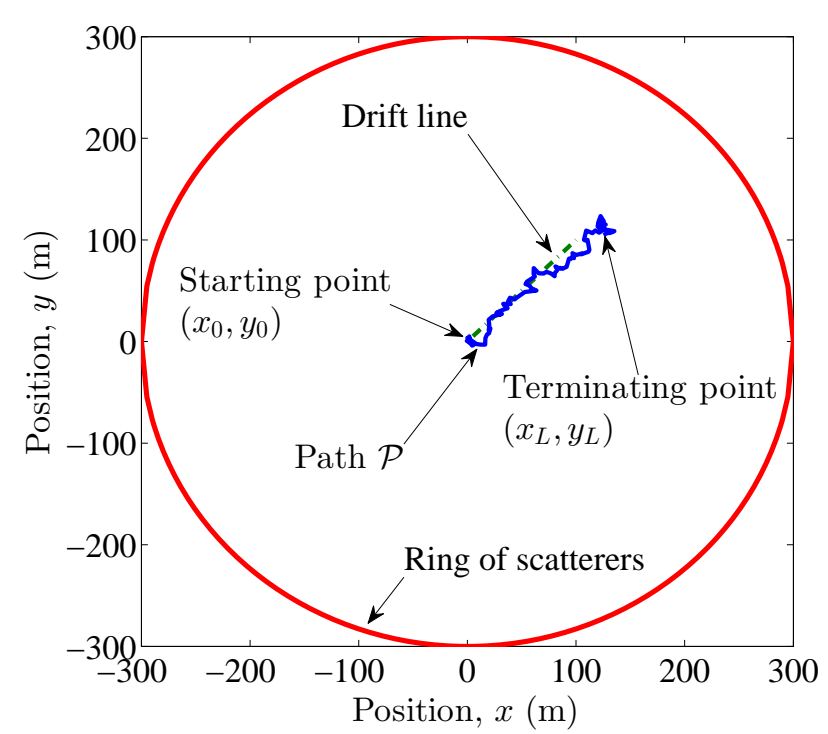

Figure 2. Realization of a targeted Brownian path $\mathcal{P}$ in the ring of scatterers. The model parameters are $k_{d}=1, L=100, \sigma_{x}=\sigma_{y}=3$, and $R=300 \mathrm{~m}$.

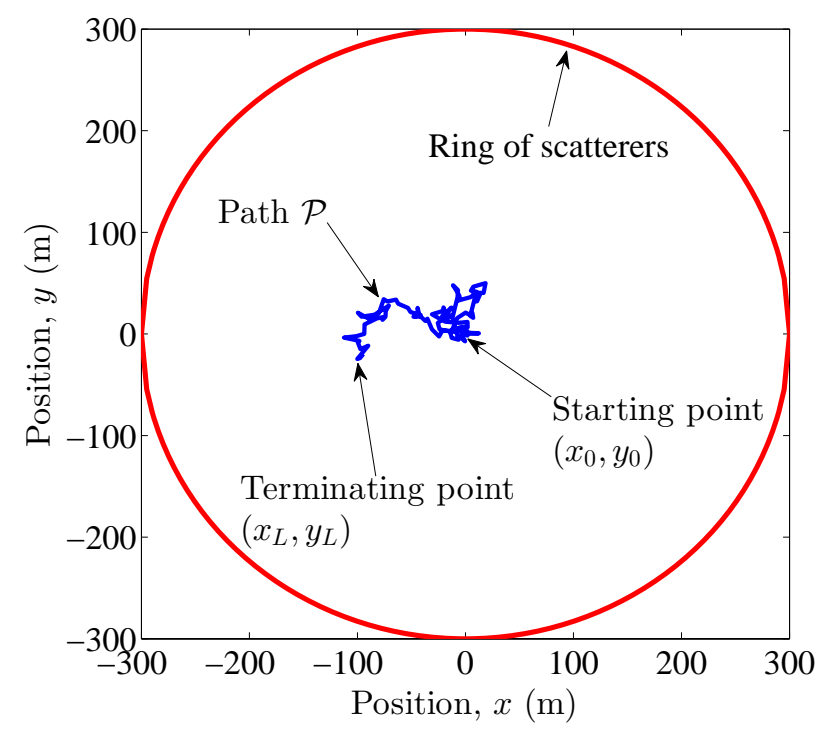

Figure 3. Realization of a non-targeted Brownian path $\mathcal{P}$ in the ring of scatterers. The model parameters are $k_{d}=0, L=100, \sigma_{x}=\sigma_{y}=6$, and $R=300 \mathrm{~m}$.

stationary non-isotropic channel model.

In what follows, we first provide an expression for the complex channel gain, and then we study the statistical characteristics of the proposed non-stationary channel model.

\section{The Complex Channel Gain}

For a typical fixed-to-mobile (F2M) scenario, the complex channel gain $\mu\left(t_{l}\right)$ of frequency-nonselective F2M channels is modeled by a complex stochastic process, representing the sum of all scattered components as follows [18]:

$$
\boldsymbol{\mu}\left(t_{l}\right)=\lim _{N \rightarrow \infty} \sum_{n=1}^{N} \boldsymbol{c}_{n} e^{j\left(2 \pi f_{n}\left(t_{l}\right) t_{l}+\boldsymbol{\theta}_{n}\right)} .
$$

Note that $\mu\left(t_{l}\right)$ accounts for the effect of nonstationarity by the time-variant Doppler frequencies ${ }^{1}$ $f_{n}\left(t_{l}\right)$ rather than the time-invariant one, i.e., $f_{n}$ (see [24, p. 59]). In this equation, $c_{n}$ stands for the attenuation factor caused by the physical interaction of the emitted wave with the $n$th scatterer $S_{n}$. In addition, the random variable $\boldsymbol{\theta}_{n}$ represents the phase shift of the $n$th path. It is assumed that $\theta_{n}$ s are independently and identically distributed random variables, each of which is uniformly distributed between $-\pi$ and $\pi$ [24, p. 59].

\section{Statistical Characteristics of the Channel Model}

We start investigating the statistical properties of the complex channel gain in (2) by deriving the local AOA at the MS, which affects strongly other statistical quantities. In addition, we study the AOM process in detail. The AOA and AOM processes will then be used to extract the PSD of the Doppler frequencies. Finally, the $\mathrm{ACF}$ of the complex channel gain is derived from the provided PSD. We remark that the illustration of the analytical results is deferred to Section 7 .

\subsection{The Local Angles-of-Arrival}

According to Figure 1, the AOA $\boldsymbol{\alpha}_{n}^{R}(l)$ at the point $\left(x_{l}, y_{l}\right)$ is computed as

$$
\boldsymbol{\alpha}_{n}^{R}(l)=\arctan \left(\frac{R \sin \left(\boldsymbol{\alpha}_{n}^{S}\right)-y_{l}}{R \cos \left(\boldsymbol{\alpha}_{n}^{S}\right)-x_{l}}\right) .
$$

For a given position index $l$, the only random variable on the right-hand side of (3) is the AOS $\boldsymbol{\alpha}_{n}^{S}$, which is assumed to be uniformly distributed between $-\pi$ and $\pi$ (see Section 4). As the number $N$ of local scatterers tends to infinity in the reference model, it is mathematically convenient to assume that the discrete AOS $\boldsymbol{\alpha}_{n}^{S}$ is a continues random variable denoted by $\boldsymbol{\alpha}^{S}$. Referring to $[18,19]$, the first-order density $p_{\alpha^{R}}\left(\alpha^{R} ; l\right)$ of the stochastic process $\alpha^{R}(l)$ in (3) is given by the following expression

$p_{\alpha^{R}}\left(\alpha^{R} ; l\right)=\frac{1}{2 \pi}\left(1-\frac{x_{l} \cos \left(\alpha^{R}\right)+y_{l} \sin \left(\alpha^{R}\right)}{\sqrt{R^{2}-\left(x_{l} \sin \left(\alpha^{R}\right)-y_{l} \cos \left(\alpha^{R}\right)\right)^{2}}}\right)$

where $-\pi \leq \alpha^{R}<\pi$. It is worth mentioning that $p_{\alpha^{R}}\left(\alpha^{R} ; l\right)$ in (4) depends heavily on the position $\left(x_{l}, y_{l}\right)$ of the MS. This means that the AOA $\alpha^{R}(l)$ is firstorder non-stationary. As a special case, if the path $\mathcal{P}$ crosses the ring's center $(0,0)$, then $p_{\alpha^{R}}\left(\alpha^{R} ; l\right)$ in (4) reduces to $1 /(2 \pi)$, which is the AOA probability density function $(\mathrm{PDF})$ of the stationary one-ring scattering model [4-6].

\footnotetext{
${ }^{1}$ The frequency shift caused by the Doppler effect is given by $f=$ $f_{\max } \cos (\boldsymbol{\alpha})$, where $f_{\max }=f_{0} v / c_{0}$ is the maximum Doppler frequency, $f_{0}$ denotes the carrier frequency, $c_{0}$ stands for the speed of light, and $\alpha$ equals the difference between the AOA and the AOM [23].
} 


\subsection{The Local Angles-of-Motion}

The path $\mathcal{P}$ can be changed to a continues and piecewise differentiable path after applying simple linear interpolation techniques. Accordingly, the AOM $\boldsymbol{\alpha}_{v}(l)$ at the point $\left(x_{l}, y_{l}\right)$ can be written in the following form

$$
\begin{aligned}
\boldsymbol{\alpha}_{v}(l) & =\arctan \left(\frac{y_{l+1}-y_{l}}{x_{l+1}-x_{l}}\right) \\
& =\arctan \left(\frac{k_{d} \Delta y+\sigma_{y}\left(B_{l+1}^{(y)}-B_{l}^{(y)}\right)}{k_{d} \Delta x+\sigma_{x}\left(B_{l+1}^{(x)}-B_{l}^{(x)}\right)}\right),
\end{aligned}
$$

where the terms $B_{l+1}^{(x)}-B_{l}^{(x)}$ and $B_{l+1}^{(y)}-B_{l}^{(y)}$ are two independent random variables of the forms $N(0, \Delta x)$ and $N(0, \Delta y)$, respectively (see Section 3.1). Therefore, the $\mathrm{AOM} \boldsymbol{\alpha}_{v}(l)$ process can be considered as the phase of a complex Gaussian process given by $s_{l}=\left(x_{l+1}-x_{l}\right)+$ $j\left(y_{l+1}-y_{l}\right)$, in which $x_{l+1}-x_{l} \sim N\left(k_{d} \Delta x, \sigma_{x}^{2} \Delta x\right)$ and $y_{l+1}-y_{l} \sim N\left(k_{d} \Delta y, \sigma_{y}^{2} \Delta y\right)$. The phase of the complex Gaussian distribution with non-identical means and variances has been studied in [25] and [26]. Herein, we avoid representing the first-order density of the AOM (the phase distribution), as it can readily be obtained by substituting the means and the variances of the underlying complex Gaussian process in [26, Eq. (2)]. As a special case, if the drift component does not exist, i.e., $k_{d}=0$, and $\sigma_{x}^{2} \Delta x=\sigma_{y}^{2} \Delta y$, then the AOM follows the uniform distribution defined over the range $(-\pi, \pi]$ (see [19]). For this special case, the AOM is first-order stationary.

\subsection{The Local Power Spectral Density}

The local Doppler frequency $f(l)$ is defined by a nonlinear transformation of the local AOA $\alpha^{R}(l)$ and the local AOM $\alpha_{v}(l)$ of the MS. It follows

$$
f(l)=f_{\max } \cos \left(\alpha^{R}(l)-\alpha_{v}(l)\right),
$$

where $\alpha^{R}(l)$ is described statistically by the first-order density $p_{\alpha^{R}}\left(\alpha^{R} ; l\right)$ in (4). Furthermore, $\alpha_{v}(l)$ describes the AOM at the point $\left(x_{l}, y_{l}\right)$ after realizing the path $\mathcal{P}$ (see (5)). Therefore, the only random variable on the right-hand side of (6) is the AOA. This is in contrast with the procedure used in [18], where the AOM is also assumed to be a random variable. To obtain the first-order density $p_{f}(f ; l)$ of the Doppler frequencies $f(l)$, we fix the position index $l$, and then we apply the concept of transformation of random variables. The result is shown in (7) (see next page), where

$$
A_{ \pm}(f ; l)=\arctan \left(\frac{y_{l+1}-y_{l}}{x_{l+1}-x_{l}}\right) \pm \arccos \left(\frac{f}{f_{\max }}\right)
$$

for $-f_{\max } \leq f \leq f_{\max }$.

With reference to [24, p. 85], the first-order density $p_{f}(f ; l)$ of the Doppler frequencies is proportional to the local PSD $S_{\mu \mu}(f ; l)$ of the complex channel gain $\boldsymbol{\mu}\left(t_{l}\right)$. This allows us to present $S_{\mu \mu}(f ; l)$ by the following expression

$$
S_{\mu \mu}(f ; l)=2 \sigma_{0}^{2} p_{f}(f ; l) .
$$

In the equation above, $2 \sigma_{0}^{2}$ is the mean power of $\mu\left(t_{l}\right)$, and $p_{f}(f ; l)$ is given by (7). For the special case that the path $\mathcal{P}$ crosses the ring's center $(0,0)$, the first-order density $p_{f}(f ; l)$ in $(7)$ reduces to

$$
p_{f}(f ; l)=\frac{1}{\pi f_{\max } \sqrt{1-\left(f / f_{\max }\right)^{2}}}
$$

which, after its multiplication by the mean power $2 \sigma_{0}^{2}$, results in the Jakes PSD [23]. Notice that the Jakes PSD is not only associated with the stationary one-ring scattering model [4-6], but also with any other scattering model that is circularly symmetric with respect to the MS [7].

\subsection{The Local Autocorrelation Function}

The local ACF $r_{\mu \mu}(\tau ; l)$ of the non-stationary complex channel gain $\mu\left(t_{l}\right)$ is obtained by taking the inverse Fourier transform of the local PSD $S_{\mu \mu}(f ; l)$ in (9) (see [27, pp. 282-285]). Accordingly, one can write

$$
r_{\mu \mu}(\tau ; l)=\int_{-f_{\max }}^{f_{\max }} S_{\mu \mu}(f ; l) e^{j 2 \pi f \tau} d f .
$$

For the special case that the path $\mathcal{P}$ goes across the ring's center, the local PSD $S_{\mu \mu}(f ; l)$ in (10) can be employed to compute the inverse Fourier transform in (11). In this case, the local ACF $r_{\mu \mu}(\tau ; l)$ in (11) reduces to $2 \sigma_{0}^{2} J_{0}\left(2 \pi f_{\max } \tau\right)$, where $J_{0}(\cdot)$ stands for the zeroth-order Bessel function of the first kind [28, Eq. (8.411.1)].

\section{Numerical Results}

We use the operating frequency $f_{0}=2.1 \mathrm{GHz}$ of UMTS in our numerical computations. To study the effect of targeted and non-targeted travelling paths of the MS, the paths $\mathcal{P}$ shown in Figures 2 and 3 have been used, respectively. Besides these two, we consider the path represented by a straight line from the starting point $(0,0)$ to the terminating point $(100,100)$. This path can be obtained either by averaging over different realizations of the targeted path shown in Figure 2, or by setting $\sigma_{x}=\sigma_{y}=0$ in (1). Given these three different paths, we illustrate the statistical properties of the channel model in terms of the AOA at the MS, PSD of the Doppler frequencies, and the ACF of the complex channel gain. It is also assumed that the MS is moving with a speed $v_{R}$ of $5 \mathrm{~km} / \mathrm{h}$, which equals an average walking speed. For the considered operating frequency, this speed allows a maximum Doppler frequency $f_{\max }$ of about $10 \mathrm{~Hz}$. The mean power $2 \sigma_{0}^{2}$ has been set to unity.

Figures 4-6 depict the first-order density $p_{\alpha^{R}}\left(\alpha^{R} ; l\right)$ of the AOA process $\alpha^{R}(l)$ given in (4). In this regard, Figure 4 shows $p_{\alpha^{R}}\left(\alpha^{R} ; l\right)$ of the AOA at the MS travelling along the targeted path $\mathcal{P}$ shown in Figure 2, while Figure 5 demonstrates that along the straight path. Furthermore, the non-targeted path shown in Figure 3 is used to display $p_{\alpha^{R}}\left(\alpha^{R} ; l\right)$ in Figure 6. A common observation in the three figures is the uniform distribution of the AOA at $l=0$, which can be attributed to the 


$$
\begin{aligned}
p_{f}(f ; l) & =\frac{1}{2 \pi f_{\max } \sqrt{1-\left(f / f_{\max }\right)^{2}}}\left(2-\frac{x_{l} \cos \left(A_{+}(f ; l)\right)+y_{l} \sin \left(A_{+}(f ; l)\right)}{\sqrt{R^{2}-\left(x_{l} \sin \left(A_{+}(f ; l)\right)-y_{l} \cos \left(A_{+}(f ; l)\right)\right)^{2}}}\right. \\
& \left.-\frac{x_{l} \cos \left(A_{-}(f ; l)\right)+y_{l} \sin \left(A_{-}(f ; l)\right)}{\sqrt{R^{2}-\left(x_{l} \sin \left(A_{-}(f ; l)\right)-y_{l} \cos \left(A_{-}(f ; l)\right)\right)^{2}}}\right)
\end{aligned}
$$

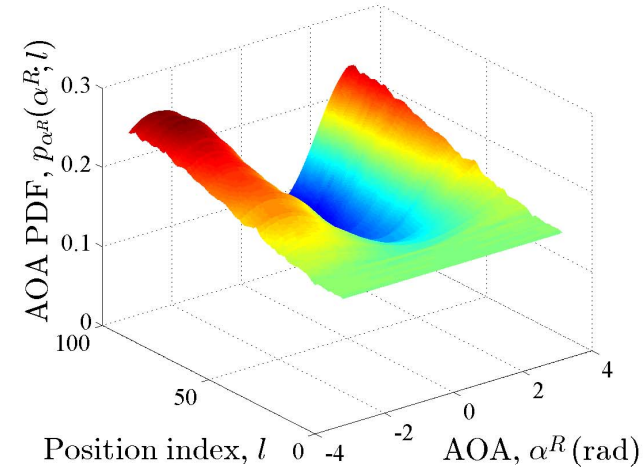

Figure 4. The behavior of the first-order density $p_{\alpha^{R}}\left(\alpha^{R} ; l\right)$ in (4) for the targeted path illustrated in Figure 2.

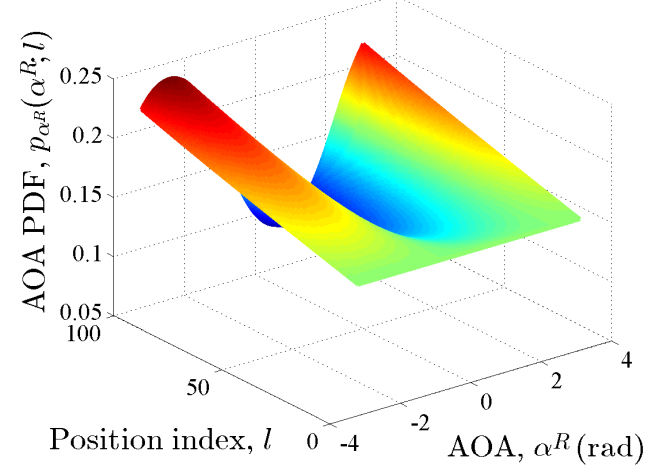

Figure 5. The behavior of the first-order density $p_{\alpha^{R}}\left(\alpha^{R} ; l\right)$ in $(4)$ for the straight path (averaged targeted path).

circularly symmetric starting point of the three paths. For the two targeted paths, the probability of receiving signals from the scatterers ahead (behind) decreases (increases) if the MS continues its motion along the path $\mathcal{P}$. However, this behaviour does not hold for the non-targeted path, in which the heading destination point is not subject to any drift. The irregular behaviour of $p_{\alpha^{R}}\left(\alpha^{R} ; l\right)$ shown in Figure 6 is indeed due to the irregular behaviour of the path shown in Figure 3. In addition, as can be observed in Figures 4 and 5, the lowest probability of receiving signals belongs to the average direction of the motion, i.e., the angle corresponds to the slope of the drift.

Figures 7-9 exhibit the local PSD $S_{\mu \mu}(f ; l)$ presented in (9). The classical Jakes PSD (resembling a U-shape) can be observed for the stationary case $(l=0)$ in all these three figures. This is again due to the circularly symmetric position of the MS at the beginning of the motion. At this position, $S_{\mu \mu}(f, 0)$ is a symmetric function with respect to $f$, justifying that the channel is isotropic at the origin. This property, however, does

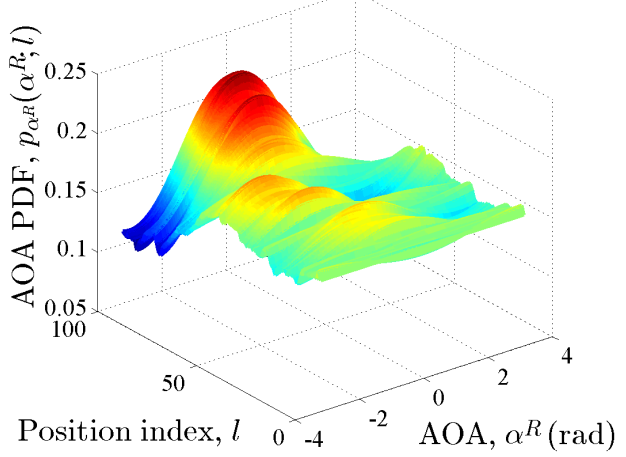

Figure 6. The behavior of the first-order density $p_{\alpha^{R}}\left(\alpha^{R} ; l\right)$ in (4) for the non-targeted path illustrated in Figure 3.

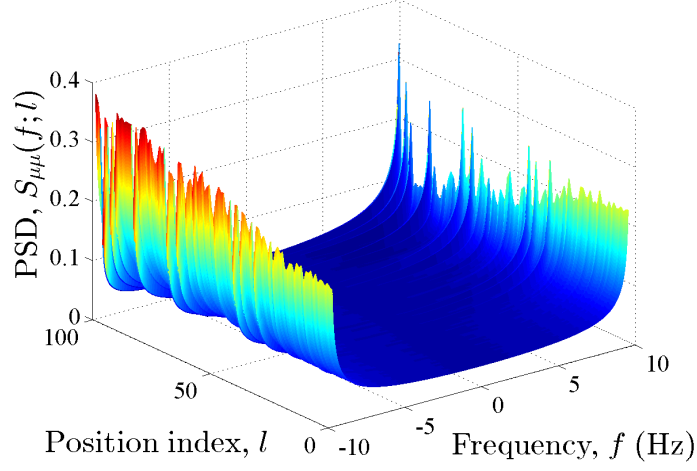

Figure 7. The behavior of the local PSD $S_{\mu \mu}(f ; l)$ in (9) for the targeted path illustrated in Figure 2.

not hold if the MS moves along any of the paths $\mathcal{P}$. The variations of the PSD in the three figures can be explained as follows. If the MS faces a lower number of scatterers ahead and a higher number of them behind, a higher probability of negative Doppler shifts can be expected. Otherwise, positive Doppler shifts occur with a higher probability. Given the two targeted paths, a higher and a lower probability of negative and positive Doppler shifts in Figures 7 and 8 can be explained by the radial drift of these paths. Nevertheless, owing to the randomness of the path, some exceptions might be observed (see Figure 7).

Figures 10-12 demonstrate the absolute value of the local ACF $r_{\mu \mu}(\tau ; l)$ provided in (11). Recalling that the PSD $S_{\mu \mu}(f ; l)$ is in general asymmetric (see Figures 79), its inverse Fourier transform, i.e., the ACF $r_{\mu \mu}(\tau ; l)$ in (11), is in general complex. Nonetheless, at the starting point $l=0$, the three figures display ACFs $\left|r_{\mu \mu}(\tau ; l)\right|$ of the form $2 \sigma_{0}^{2}\left|J_{0}\left(2 \pi f_{\max } \tau\right)\right|$. With reference to Figures 10-12, the ACF varies in position (time), justifying that the proposed channel model is non- 


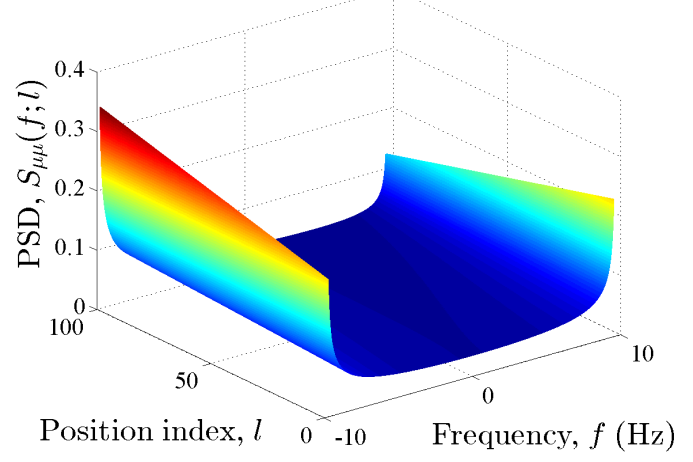

Figure 8. The behavior of the local PSD $S_{\mu \mu}(f ; l)$ in (9) for straight path (averaged targeted path).

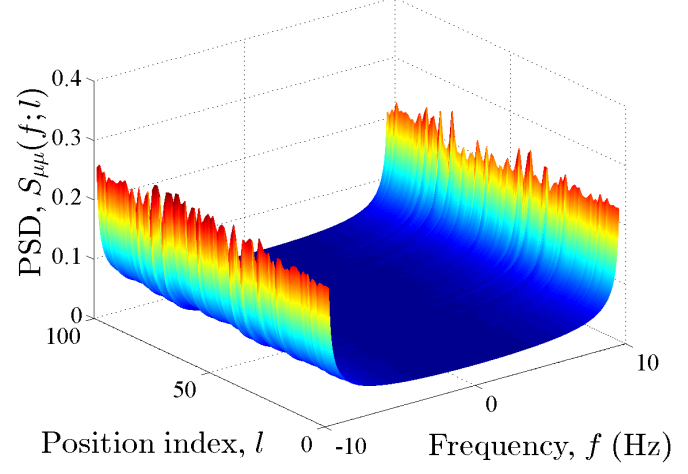

Figure 9. The behavior of the local PSD $S_{\mu \mu}(f ; l)$ in (9) for the nontargeted path illustrated in Figure 3.

stationary. Referring to Figures 10 and 11, the correlation grows by increasing $l$ for a given value of $\tau \neq 0$. This growth, however, occurs with some fluctuations (see Figure 10), which are due to the randomness of the corresponding path (see Figure 2). We finish this part by remarking that herein, the effect of changing motion patterns (path models) can better be observed (described) by plotting the PSD rather than the ACF.

\section{Conclusion}

This paper has proposed a Brownian path model to generate realistic trajectories of the MS. Particularity, the proposed path model unifies both targeted and nontargeted motions of the MS. We have employed the proposed path model to provide a non-stationary channel model. To cope with the scattering effect, we have utilized the non-centred one-ring scattering model, in which the MS is surrounded by a ring of scatterers centred not necessarily on the MS. By tracking the MS on the proposed Brownian path, we have derived analytical expressions for the time-varying AOA and AOM. The first-order densities of $\mathrm{AOA}$ and $\mathrm{AOM}$ processes have been computed. These processes have then been used to compute the PSD of the Doppler frequencies and the ACF of the complex channel gain. It has been shown that the proposed path model results in a nonstationary non-isotropic channel model. Nevertheless, the stationary isotropic one-ring scattering model can be obtained as a special case. Considering a walking

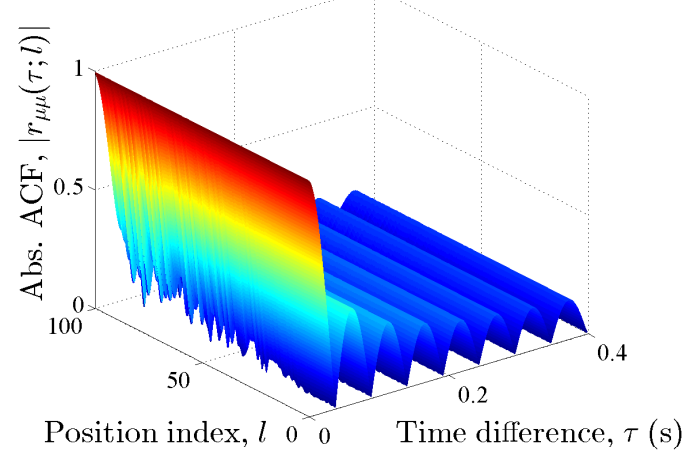

Figure 10. The behavior of the absolute value of the local ACF $\left|r_{\mu \mu}(\tau ; l)\right|$ (see (11)) for the targeted path illustrated in Figure 2.

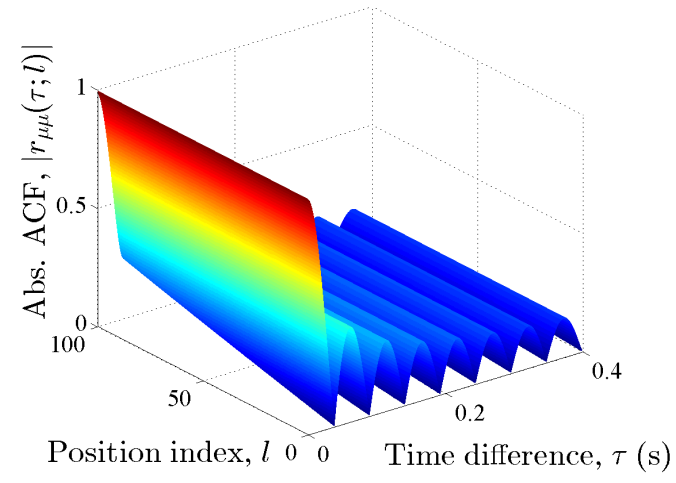

Figure 11. The behavior of the absolute value of the local ACF $\left|r_{\mu \mu}(\tau ; l)\right|$ (see (11)) for the straight path (averaged targeted path).

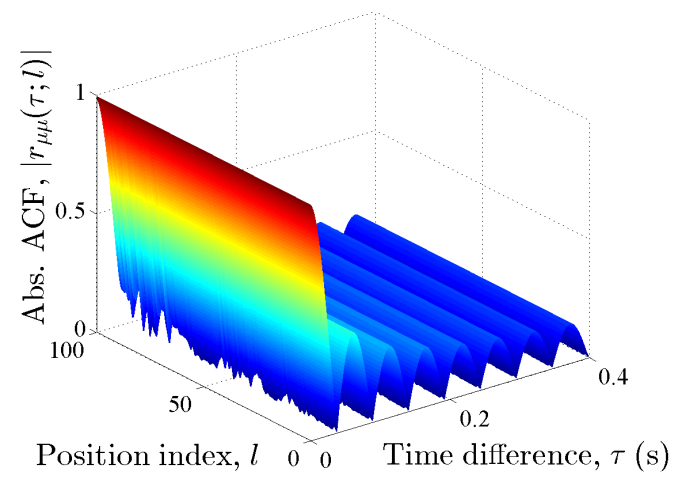

Figure 12. The behavior of the absolute value of the local ACF $\left|r_{\mu \mu}(\tau ; l)\right|$ (see (11)) for the non-targeted path illustrated in Figure 3.

speed scenario, we have illustrated the statistical characteristics of the proposed channel model. It has been shown that the AOA and AOM processes are in general first-order non-stationary, which implies that the PSD and ACF depend on time. We have also shown that the effect of changing mobility pattern can better be seen in the PSD as compared to the ACF of the complex channel gain. In future works, the analytical results will be verified by means of empirical data.

\section{REFERENCES}

[1] J. Karedal, F. Tufvesson, N. Czink, A. Paier, C. Dumard, T. Zemen, C. Mecklenbräuker, and A. Molisch, "A geometry-based stochastic MIMO model for vehicle-to- 
vehicle communications," IEEE Transactions on Wireless Communications, vol. 8, no. 7, pp. 3646-3657, Jul. 2009.

[2] A. F. Molisch, A. Kuchar, J. Laurila, K. Hugl, and R. Schmalenberger, "Geometry-based directional model for mobile radio channels - principles and implementation," European Transactions on Telecommunications, vol. 14, no. 4, pp. 351-359, 2003.

[3] A. F. Molisch, "A generic model for MIMO wireless propagation channels in macro- and microcells," IEEE Transactions on Signal Processing, vol. 52, no. 1, pp. 61-71, 2004.

[4] D.-S. Shiu, G. J. Foschini, M. J. Gans, and J. M. Kahn, "Fading correlation and its effect on the capacity of multielement antenna systems," IEEE Transactions on Communication Technology, vol. 48, no. 3, pp. 502-513, Mar. 2000.

[5] A. Abdi and M. Kaveh, "A space-time correlation model for multielement antenna systems in mobile fading channels," IEEE Journal of Selected Areas in Communications, vol. 20, no. 3, pp. 550-560, Apr. 2002.

[6] M. Pätzold and B. O. Hogstad, "A space-time channel simulator for MIMO channels based on the geometrical one-ring scattering model," in Proc. 60th IEEE Semiannual Veh. Technol. Conf., VTC 2004-Fall, vol. 1. Los Angeles, CA, USA, Sep. 2004, pp. 144-149.

[7] A. Borhani and M. Pätzold, "A unified disk scattering model and its angle-of-departure and time-of-arrival statistics," IEEE Transactions on Vehicular Communications, vol. 62, no. 2, pp. 473-485, Feb. 2013.

[8] K. T. Wong, Y. I. Wu, and M. Abdulla, "Landmobile radiowave multipaths' DOA-distribution: Assessing geometric models by the open literature's empirical datasets," IEEE Transactions on Antennas and Propagation, vol. 58, no. 2, pp. 946-958, Feb. 2010.

[9] A. Ispas, G. Ascheid, C. Schneider, and R. Thom, "Analysis of local quasi-stationarity regions in an urban macrocell scenario," in Proc. 71th IEEE Vehicular Technology Conference, VTC 2010-Spring. Taipei, Taiwan, May 2010.

[10] A. Gehring, M. Steinbauer, I. Gaspard, and M. Grigat, "Empirical channel stationarity in urban environments," in Proc. 4th European Personal Mobile Communications Conference. Vienna, Austria, Feb. 2001.

[11] D. Umansky and M. Pätzold, "Stationarity test for wireless communication channels," in Proc. IEEE Global Communications Conference, IEEE GLOBECOM 2009. Honolulu, Hawaii, USA, Nov./Dec. 2009.

[12] A. Paier, J. Karedal, N. Czink, H. Hofstetter, C. Dumard, T. Zemen, F. Tufvesson, A. F. Molisch, and C. F. Mecklenbräucker, "Characterization of vehicle-to-vehicle radio channels from measurement at $5.2 \mathrm{GHz}$," Wireless Personal Communications (WPC), vol. 50, no. 1, pp. 19-32, Jul. 2009.

[13] G. Matz, "On non-WSSUS wireless fading channels," IEEE Transactions on Wireless Communications, pp. 24652478, Sep. 2005.

[14] A. Ghazal, C. Wang, H. Hass, R. Mesleh, D. Yuan, and X. Ge, "A non-stationary MIMO channel model for high-speed train communication systems," in Proc. 75th IEEE Vehicular Technology Conference, VTC 2012-Spring. Yokohama, Japan, May 2012.

[15] A. Chelli and M. Pätzold, "A non-stationary MIMO vehicle-to-vehicle channel model based on the geometrical T-junction model," in Proc. International Conference on Wireless Communications and Signal Processing, WCSP 2009. Nanjing, China, Nov. 2009.

[16] J. Karedal, F. Tufvesson, N. Czink, A. Paier, C. Dumard, T. Zemen, C. F. Mecklenbräuker, and A. F. Molisch, "A geometry-based stochastic MIMO model for vehicle-tovehicle communications," IEEE Transactions on Wireless Communications, vol. 8, no. 7, pp. 3646-3657, Jul. 2009.

[17] A. Borhani and M. Pätzold, "A non-stationary one-ring scattering model," in Proc. IEEE Wireless Commun. and Net. Conf. (WCNC'13). Shanghai, China, Apr. 2013.
[18] _ "Modelling of non-stationary mobile radio channels incorporating the Brownian mobility model with drift," in Lecture Notes in Engineering and Computer Science: World Congress on Engineering and Computer Science, WCECS'13, vol. 2. San Francisco, USA, Oct. 2013, pp. 695-700.

[19] — "Modelling of non-stationary mobile radio channels using two-dimensional Brownian motion processes," in Proc. 6th International Conference on Advanced Technologies for Communications, ATC'13. Ho Chi Minh City, Vietnam, Oct. 2013, pp. 241-246.

[20] R. C. Earnshaw and E. M. Riley, "Brownian motion: Theory, modelling and applications," 2011.

[21] T. Camp, J. Boleng, and V. Davies, "A survey of mobility models for ad hoc network research," Wireless Communications and Mobile Computing, vol. 2, no. 5, pp. 483-502, Sep. 2002.

[22] B. H. Fleury and D. Dahlhaus, "Investigations on the time variations of the wide-band radio channel for random receiver movements," in Proc. IEEE International Symposium on Spread Spectrum Techniques and Applications (ISSSTA'94), vol. 2. Finland, Oulu, 1994, pp. 631-636.

[23] W. C. Jakes, Ed., Microwave Mobile Communications. Piscataway, NJ: IEEE Press, 1994.

[24] M. Pätzold, Mobile Fading Channels, 2nd ed. Chichester: John Wiley \& Sons, 2011.

[25] V. A. Aalo, G. P. Efthymoglou, and C. Chayawan, "On the envelope and phase distributions for correlated Gaussian quadratures," IEEE Communications Letters, vol. 11, no. 12, pp. 985-987, 2007.

[26] P. Dharmawansa, N. Rajatheva, and C. Tellambura, "Envelope and phase distribution of two correlated Gaussian variables," IEEE Transactions on Communications, vol. 57, no. 4, pp. 915-921, 2009.

[27] F. Hlawatsch and F. Auger, Time-Frequency Analysis: Concepts and Methods. London (UK): ISTE and Wiley, 2008.

[28] I. S. Gradshteyn and I. M. Ryzhik, Table of Integrals, Series, and Products, 7th ed. Elsevier Academic Press, 2007.

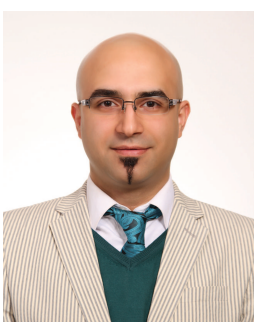

Alireza Borhani received the B.E. degree in biomedical engineering from Azad University, Tehran, Iran, in 2007 and the M.E. degree in telecommunication engineering from Shahed University, Tehran. He is currently working toward the Ph.D. degree at the Department of Information and Communication Technology, University of Agder, Grimstad, Norway.

His research interests include mobile radio communications, especially multipath fading channel modelling, multiple-input multipleoutput (MIMO) systems, vehicular-to-vehicular communications, and information theory.

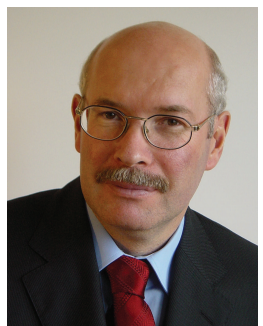

Matthias Pätzold received the Dipl.-Ing. and Dr.-Ing. degrees in electrical engineering from Ruhr-University Bochum, Bochum, Germany, in 1985 and 1989, respectively, and the habil. degree in communications engineering from the Technical University of HamburgHarburg, Germany, in 1998. From 1990 to 1992, he was with ANT Nachrichtentechnik $\mathrm{GmbH}$, Backnang, Germany, where he was engaged in digital satellite communications. From 1992 to 2001, he was with the department of digital networks at the Technical University HamburgHarburg. Since 2001, he has been a full professor of mobile communications with the University of Agder, Norway. He authored several books and numerous technical papers. His publications received ten best paper awards. He has been actively participating in numerous conferences serving as TPC chair and TPC member. 